\title{
Process Control System Considering the Machines Functional Flexibilities
}

\author{
Osvaldo L. Asato, Guilherme M. Dobrianskyj, \\ Fabrício Junqueira, Diolino J. Santos Filho, and Paulo E. Miyagi \\ Escola Politécnica da Universidade de São Paulo, Av.Prof. Mello Moraes, 2231, Brazil \\ \{osvaldo.asato, fabri, diolinos, pemiyagi\} @usp.br
}

\begin{abstract}
The evolution of tool-machines shows a trend for integration of different machining functionalities such as, milling, turning, and drilling, on a same machine. These machines are result of the mechatronics development that allows the aggregation of different functionalities into a unique machine (named multifunctional machine tool - MMT). However from the point of view of the production process there is a limitation of the conventional process control solution, i.e., when the systems are composed by MMTs, their functional flexibilities are considered only during scheduling and encapsulated at this level, and during the process execution, MMTs are viewed as traditional ones. Then, the multi-functionalities are not explored for resources allocation. Thus, a new process control approach is proposed here to dynamically allocate the resources exploring the functional flexibilities of MMTs.
\end{abstract}

Keywords: Multifunctional Machine Tools, Functional flexibility, Petri net, Ant Colony Optimization.

\section{Introduction}

The technological developments in mechatronics systems have allowed the aggregation of several machining functionalities (such as milling, turning and drilling) at a same machine tool [6]. These multifunctional machine tools (MMTs) have then a large functional flexibility than conventional machine tools. These MMTs potentially meet the need for productivity and efficiency of production processes, especially in the production of customized products [1], [9]. However, currently the process control solutions for productive systems (PSs) do not consider and do not explore any nondeterministic behavior that the MMTs flexibility introduces in these systems. Conventionally, MMTs are used as a type of machining center [10], where the focus is on the centralization of the functionalities in the same machine (without work piece manipulation between different machines). In this context, a deterministic (static) allocation of resources (machine tools, machining centers, MMTs) is adopted according to the production plan [3], [4], and in the case of intrinsic indeterminism, such as delays or early finish of production activities, the potential flexibility of MMTs are not used to maintain or improve the PS's global performance. 
Therefore, to explore the functional flexibility of MMTs, a new process control solution must be considered. The focus must be on both functional and operational flexibilities of the MMTs when the resources are designated in real time according to the functionality demand from the production process. This approach changes the paradigm of the resource allocation control that conventionally is based on the pre-defined functionality of the available resources. The proposed control solution for PS considers the necessary functionalities for the execution of each activity of production process, and establishes a dynamic allocation of resources. The adopted modeling tool considers that production processes and its control can be treated as a class of discrete event system [2], [5], [7], [8]. This paper is organized as follows: Contribution to value creation in section 2; Fundamental concepts in section 3; Process Control with functional flexibility in section 4; Case study in section 5 and the Conclusion in section 6.

\section{Contribution to Value Creation}

Although the technological evolution has developed resources with multiple functionalities as MMTs, it wasn't developed a control system that, at the moment of the allocation of the resource the shop floor, which of the functionalities of this machine is the most appropriate to use in relation the global production process.

In current Resource Allocation Control Systems (RACS) for PS, MMTs are used as conventional machine, because the control system works with static allocation, in other words, the functionalities are attributed for a local processing in such a way that RACS doesn't have access to this type of information. In this context, the current concept of RACS doesn't explore the potentiality of MMTs, once the same was not projected to allocate functionalities. It is observed that the solutions of RACS use graphs of allocation of resources [8], but there are not proposals in the sense of building, in equivalent way, graph of allocation of functionalities. In fact to explore the potentiality of MMTs is necessary to modify the structure of RACS, due to emergence of one more variable control called functionality. In the conventional control systems there were a one to one relationship between resource and functionality, sparing additional control strategies. However, if this reality was modified in which a resource possess a group of associated functionality, is necessary to establish specifics control rules to treat this new variable. Taking in account a new paradigm is proposed in terms of modular structure for a in that the control of allocation of functionalities to the resources in real time it is modeled to improve the efficiency of the global system [12]. The term efficiency should be understood as smaller cost for production: (i) it can decrease the time of execution; (ii) it can avoid that the system enters in deadlock for not being capable of reconfiguring the plan of the allocation of resources.

The obtained result will be: (i) to perfect the rational use of the resources in productive systems; (ii) to increase the competitiveness among the companies; (iii) to generate benefits for the society that can have access to products of lower cost.

\section{Fundamental Concepts}

The production process as well as its control procedure can be treated as a processes with discrete states that are abruptly changed when an instantaneous event occurs, for example machine that no working. This process defines a class of discrete event 
system [2], [5], [7], [8] and therefore, modeling tools such as Petri net [2] can be used not only to describe the structure and behavior of the processes, but also as a specification of a desired task execution.

Based on previous works related with PSs, the Enhanced Mark Flow Graph (EMFG) [7] is adopted to model the production processes, and to specify its control solution. The E-MFG is derived from Petri net considering individualized marks through the association of attributes. With E-MFG is possible to control the information flow in the model, i.e., modifying the attributes of the marks and, based on this information, designate resource using an element denominated control-box. In this context, the control-box works with ant colony algorithm to designate the machine in the shop floor.

\subsection{Enhanced - Mark Flow Graph (E-MFG)}

The E-MFG is effective to model control strategies, especially when associated to the concept of "functional blocks" for modular and distributed control as established in the standard IEC 61499. From the practical point of view of SPs, marks in E-MFG are associated to a vector of attributes that guarantees their individuality. These attributes can be associated to the information about products, processes, transportation resources, transformation resources etc.. The manipulation of the mark's attributes indicates the alteration of some information that is interpreted as an updating of the local states of the system (state associated with a box that represents an activity, operation or task of the production process). The control-box accomplishes this function based on ant colony algorithm that is applied for the verification and update the attributes previously specified, and also according to the firing rule of the transitions, as exemplified in Fig. 1. In this example, a mark with the attributes $<\mathrm{a} 1, \mathrm{a} 2, \mathrm{a} 3, \mathrm{a} 4>$ where: a1=functionality, a2 = type of work-piece, a3= origin (from where the work-piece is coming from), a4= allocation (where the piece should go).In PS, there is an intense interaction among the entities that compose the system [5] and E-MFG has also has a specific graph structures called transmission and reception interface [8].

The transmission interface sends an asynchronous message when the respective box is marked. The transmission interface corresponds to the "method call" of an "object" based on the object-oriented paradigm. This structure models the message emission for a system and it is composed by a send transition and a send arc (Fig. 2a).

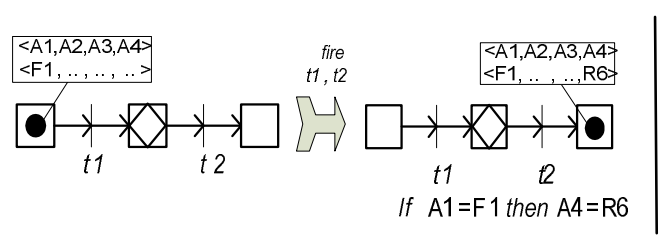

Fig. 1. E-MFG with transitions firing

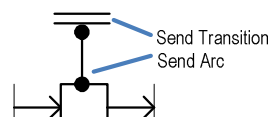

(a) Interface of send

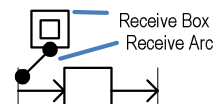

b) Interface of reception

Fig. 2. Example of communicators 
The reception interface enables or inhibits the transition firing connected to it, in order to the received information. It models the capture and the processing of messages coming from other systems. This structure is composed of a receive box and a receive arc (Fig. 2b).

\subsection{Algorithm Ant Colony Optimization}

The algorithm "ant colony optimization" (ACO) was developed by Dorigo [11] and in his work; this algorithm was used to analyze the problem of resource designation. In this context the ACO verifies which resources have the functionality required and among them the resource that has a functionality with lower operating cost is selected.

The state transition rule is as follows:

i. $\quad$ an ant positioned on node $r$ chooses the resource $s$ to move to by applying the rule given by Eq. (1).

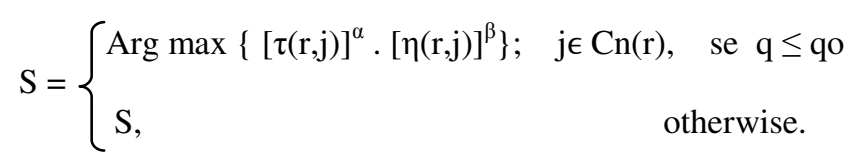

Where: $S$ is the next resource, $r=$ previous resource, $n=$ total number of resources, $C n(r)=$ group of the resources that possess the following functionality, $S=$ defined resource following the distribution of probability $p(r, s)$.

ii. $\quad \mathrm{P}(\mathrm{r}, \mathrm{s})$ probability for an ant to travel from node $\mathrm{r}$ to node $\mathrm{s}$, the rule given by Eq.(2);

$$
\mathrm{P}(\mathrm{r}, \mathrm{s})= \begin{cases}\frac{[\tau(\mathrm{r}, \mathrm{s})]^{\alpha} \cdot[\eta(\mathrm{r}, \mathrm{s})]^{\beta}}{\sum_{\mathrm{i}} \epsilon_{\mathrm{cn}(\mathrm{r})}[\tau(\mathrm{r}, \mathrm{i})]^{\alpha} \cdot[\eta(\mathrm{r}, \mathrm{i})]^{\beta}} & \text { if } \mathrm{s} \in \mathrm{Cn}(\mathrm{r}) \\ 0 & \text { otherwise }\end{cases}
$$

Where: $n=$ total number of resources, $C n(r)=$ group of the resources that possess the following functionality.

iii. Pheromone level based on local updating rule: $\tau(\mathrm{r}, \mathrm{s}) \leftarrow(1-\rho) . \tau(\mathrm{r}, \mathrm{s})$

iv. Pheromone level based on global updating rule: $\tau(\mathrm{r}, \mathrm{s}) \leftarrow(1-\alpha) \cdot \tau(\mathrm{r}, \mathrm{s})+\alpha \cdot \Delta \tau(\mathrm{r}, \mathrm{s})$

\section{$4 \quad$ Process Control with Functional Flexibility}

The approach adopted is based on the semantic division of the information considered for the production process control: (a) the information related to functionalities requested to execute an activity, and (b) the information related to allocation of 
resources in accordance with the functionality requested. This approach allows resources to be designated in real time according to the functionality demand.

\subsection{RACS Structure}

In this work is proposed an evolution [12] according to Fig. 3a where exists: (i) a specific module for production sequence control (PsC); (ii) a module for functionality designation (FD) according to the set of activities to be executed; and (iii) a module for resources designation based on the functionalities (RD-F). Each module accomplishes specific control functions and, through the cooperation among them, production activities (in accordance with a global production plan) are executed, as described to proceed:

- PsC represents the sequence of operations to be executed for the production of one product. This control module needs to request resources, and then receives information about the designation of resources to be allocated for the execution of the set of the operations in subject.

- The FD controls module identifies the functionality associated with the requested resources from PsC, according to the original production plan. This module specifies the functionality corresponding to the resources requested by the PsC.

- The RD-F controls module designates the resources, i.e., RD-F determines the association between functionality and the best resource to effectively execute each process operation.

\subsection{RD-F Algorithm}

The resource designation is made based on ACO that is processed whenever there is a transition of states in the FD control module: the RD-F module considers the actual state of each machine including MMTs, and also its operational and functional flexibility. These modules interact in the following way: (i) $\mathrm{PsC}$ monitors the evolution of the production processes and when a new activity must be executed, it sends a request to FD about the needed resources according to the original production plan; (ii) based on this request, FD identifies the respective functionality and sent this information to RD-F that accomplishes the designation of the resource that can execute the requested functionality, according to ACO: the solution corresponds to the vector of Resources that possess the largest pheromone. This solution is sent back for $\mathrm{PsC}$; (iii) Once this information is received, $\mathrm{PsC}$ allocates the specified resource, and control the execution of the activity production.

\subsection{Synthesis of the Control Modules}

The control solution, i.e., the procedures to be executed in each module of RACS can be specified through E-MFG models and ACO algorithm. Fig. 4 illustrates the procedure to develop the models used for decision making. 


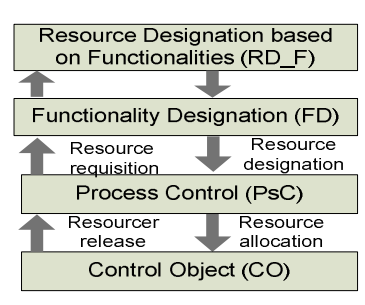

Fig. 3a. Modules of the control system

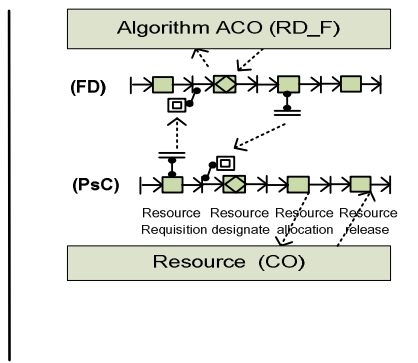

Fig. $3 b$. com
modules

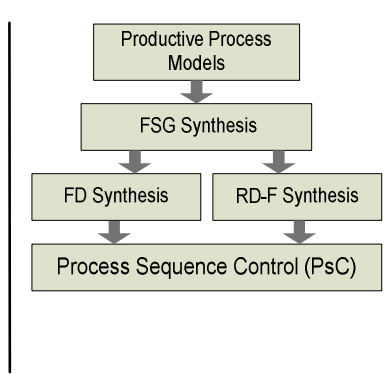

Fig. 4. Procedure to synthesis the models

The procedure is composed of the following steps:

Step 1 - FSG Synthesis. Based on each production process model, a general structure of "functionalities sequence graph" (FSG) is developed in E-MFG. Each resource is mapped according to the functionality that is necessary to execute the corresponding activity (see Fig. 4).

Step 2 - Refinement of FSG. For each activity of the production process, FSG identifies a specific functionality. The information about the resource can be encapsulated through the concept of individual marks of the E-MFG. Therefore, the vector of mark attributes is defined as: $<\mathrm{a} 1, \mathrm{a} 2>$ where, a1=Fi (functionality Fi to execute an activity of the production process) and a2=Ri (resource $\mathrm{Ri}$ according to the production process model previously specified). In this context, the graph structure between transitions $\mathrm{Ai}$ and $\mathrm{Ai}+1$ of FSG is refined trough a graph with a box (input buffer), a control-box (representing the functionality) and a box (exit buffer) (Fig. 5). The rules associated with the control-box will be defined in step 4 .

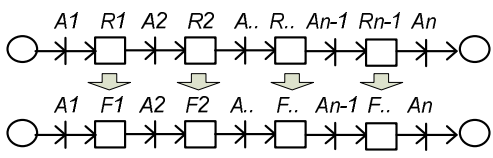

Fig. 5. Control model for FSG



Fig. 6. Refinement of control model for FSG

Step 3 - FD Synthesis. From refined FSG of each production process, the elements with the same semantics are identified and merged, ie, control-boxes related to the same functionality are fused. The result E-MFG global model of all refined FSG is the control model for FD module. The problem of conflict when there is dispute of resources for a same functionality is solved automatically looking for the solution of smaller global cost according to ACO.

Step 4 - RD-F Rules Synthesis. In this step, the ACO is applied to generate the best designation of resources (lower operation cost) whenever there is a state transition in FD. The cost table associated to ACO is based on the information defined previously in the scheduling phase. Therefore, the rule for resources designation is executed 
based on ACO and the solution of the algorithm is used to update the mark's attributes of the E-MFG model of FD.

Step 5 - PsC Synthesis. It is derived from control models for FSG as is it follows:

- It is added a complementary box related to each box Fi of the FSG models.

- Each box Fi between transitions $\mathrm{Ai}$ and $\mathrm{Ai}+1$ of FSG is substituted by a graph with: (i) a box to model the request of a resource, (ii) a box to represent the allocation of a resource and (iii) a box to indicate that the resource is available.

- The interaction between PsC, FD and RD-F modules are established through communicator arcs (send and receive arcs) Fig. 3 b.

\section{Case Study}

Consider a PS that has a production process with:

- Functionalities F1, F2, F3.

- Machines R1, R2, R3, R4, R5, R6. Each resource has the functionalities according to Table 1 .

Table 1. Description of the functionalities of each resource of the PS

\begin{tabular}{|c|c|c|c|c|c|}
\hline Resource & functionality & Resource & functionality & Resource & functionality \\
\hline R1 & F1 & R3 & F2 & R5 & F2 and F3 \\
\hline R2 & F1 & R4 & F2 & R6 & F2 and F3 \\
\hline
\end{tabular}

Hypothesis:

- All machines are available at time $\mathrm{t}=0$. The machines do not break.

- The transportation time between machines is not considered

- The setup time is not considered for all machines.

The production process has the following the sequence: $\mathrm{F} 1 \rightarrow \mathrm{F} 2 \rightarrow \mathrm{F} 3$.

Taking in account the worst case is when the functionalities are being requested simultaneously. The Table 2 represents the costs associated, and the first column (resources R1, R2, R3, R4, R5, R6) refers to the machine of origin (start) and the first line refers to the machine of destiny (next machine allocated).

When beginning the process in one of the machines, an initial cost exists (start of the process) according to Table 2.

Table 2. The initial cost attributed to the resources

\begin{tabular}{|l|l|l|l|l|l|}
\hline $\mathrm{R} 1=2.5$ & $\mathrm{R} 2=2$ & $\mathrm{R} 3=3$ & $\mathrm{R} 4=10$ & $\mathrm{R} 5=7$ & $\mathrm{R} 6=6$ \\
\hline
\end{tabular}

In same way for the last machine Table 3.

Table 3. The end cost attributed to the resources

\begin{tabular}{|l|l|l|l|l|l|}
\hline $\mathrm{R} 1=10$ & $\mathrm{R} 2=9$ & $\mathrm{R} 3=3$ & $\mathrm{R} 4=4$ & $\mathrm{R} 5=2$ & $\mathrm{R} 6=5$ \\
\hline
\end{tabular}


Table 4. Table Cost associated to the resources

\begin{tabular}{|c|c|c|c|c|c|c|}
\hline From ${ }^{\text {To }}$ & R1 & $\mathbf{R 2}$ & $\mathbf{R 3}$ & R4 & R5 & R6 \\
\hline R1 & 1 & 3 & 2 & 8 & 4 & 5 \\
\hline $\mathrm{R} 2$ & 3 & 1 & 5 & 13 & 8 & 2 \\
\hline R3 & 3 & 4 & 1 & 7 & 4 & 11 \\
\hline R4 & 10 & 8 & 7 & 1 & 5 & 3 \\
\hline R5 & 7 & 8 & 1 & 5 & 1 & 4 \\
\hline R6 & 1 & 3 & 2 & 7 & 3 & 1 \\
\hline
\end{tabular}

Table 5. Obtained solution using algorithm based on ACO

\begin{tabular}{|c|c|c|c|c|c|c|c|c|c|}
\hline Ant & F1 & F2 & F3 & Cost & Ant & F1 & F2 & F3 & Cost \\
\hline 1 & R2 & R6 & R6 & 10 & 27 & R2 & R6 & R6 & 10 \\
\hline 2 & $\mathrm{R} 1$ & R3 & R5 & 10,5 & 28 & $\mathrm{R} 1$ & R3 & R5 & 10,5 \\
\hline 3 & $\mathrm{R} 2$ & R3 & R6 & 23 & 29 & $\mathrm{R} 2$ & R6 & R6 & 10 \\
\hline 4 & R1 & R5 & R5 & 9,5 & 30 & R1 & R3 & R6 & 15,5 \\
\hline 5 & R2 & R6 & R5 & 9 & 31 & R2 & R6 & R5 & 9 \\
\hline 6 & $\mathrm{R} 1$ & R4 & R6 & 18,5 & 32 & R2 & R6 & R5 & 9 \\
\hline 7 & R2 & R4 & R5 & 22 & 33 & R2 & R6 & R5 & 9 \\
\hline 8 & R1 & R6 & R6 & 13,5 & 34 & R2 & R6 & R5 & 9 \\
\hline 9 & R2 & R5 & R6 & 19 & 35 & R2 & R3 & R5 & 13 \\
\hline 10 & R1 & R6 & R5 & 12,5 & 36 & R2 & R6 & R6 & 10 \\
\hline 11 & $\mathrm{R} 2$ & R6 & R5 & 9 & 37 & $\mathrm{R} 2$ & R4 & R5 & 22 \\
\hline 12 & R2 & R6 & R6 & 10 & 38 & R2 & R3 & R5 & 13 \\
\hline 13 & R2 & R3 & R5 & 13 & 39 & R2 & R5 & R6 & 19 \\
\hline 14 & $\mathrm{R} 2$ & R5 & R5 & 13 & 40 & R1 & R6 & R5 & 12,5 \\
\hline 15 & R2 & R4 & R5 & 22 & 41 & R2 & R6 & R5 & 9 \\
\hline 16 & R2 & R6 & R5 & 9 & 42 & $\mathrm{R} 2$ & R6 & R5 & 9 \\
\hline 17 & R1 & $\mathrm{R} 4$ & R6 & 18,5 & 43 & $\mathrm{R} 2$ & R6 & R5 & 9 \\
\hline 18 & R2 & R3 & R6 & 23 & 44 & $\mathrm{R} 2$ & R6 & R5 & 9 \\
\hline 19 & $\mathrm{R} 1$ & R5 & R5 & 9,5 & 45 & $\mathrm{R} 2$ & R6 & R5 & 9 \\
\hline 20 & R2 & R6 & R6 & 10 & 46 & R2 & R5 & R5 & 13 \\
\hline 21 & $\mathrm{R} 2$ & R6 & R5 & 9 & 47 & $\mathrm{R} 2$ & R4 & R6 & 23 \\
\hline 22 & $\mathrm{R} 2$ & R6 & R5 & 9 & 48 & $\mathrm{R} 2$ & R4 & R6 & 23 \\
\hline 23 & R2 & R5 & R6 & 19 & 49 & R2 & R4 & R5 & 22 \\
\hline 24 & R2 & R6 & R5 & 9 & $\mathbf{5 0}$ & R2 & R3 & R6 & 23 \\
\hline 25 & R2 & $\mathrm{R} 4$ & R6 & 23 & & & & & \\
\hline 26 & R2 & R5 & R5 & 13 & & & & & \\
\hline
\end{tabular}


When processing the ACO algorithm was considered:

- $\quad$ The following parameters: $\alpha=1 ; \quad \beta=2 ; \quad \rho=0.9 ; \quad \phi=0.2 ; \quad$ qo $=0.5$.

- Initial value of the pheromone $=0.3$.

- Number of iterations: 50 (with 10 ants). The result is presented in the Table 5.

The solution is: R2 - R6 - R5.

\section{Conclusion}

This approach uses algorithm ACO, for each scenario were performed 50 iterations on Core 2 Quad CPU Q9550 2.84Ghz 4GB of RAM and Windows 7 system. In this condition the algorithm takes 16 milliseconds to meet the result (R2, R6, R5) with a cost 9 units. Therefore, the proposed approach for the production process control system using functionality allocation makes possible the dynamic allocation of resources in agreement with the requested functionality of the process. This aspect is fundamental to explore the potential of production systems (PSs) with multifunctional machine tools (MMTs).

The process control system proposed in this work can dynamically make decisions in accordance with the monitored signals of the PS exploring the flexibility of the available resources when activity delays of early finishing occurs. These are the first result using ACO. The following next researches are about the use of this approach to avoid deadlock in complex PS.

Acknowledgments. The authors thank the financial support of the Brazilian government agencies, CNPq, FAPESP and to the MEC/CAPES/PET program.

\section{References}

1. Aronson, R.B.: Machine-tools basics part1. Manufacturing Engineering 126(3), 54-56 (2001)

2. Cangea, O.: Designing a control structure for discrete event systems described by Petri nets. In: Annals of DAAM and Proc. (2010)

3. He, L.: Research on job shop scheduling aiming at instant customerisation. In: Proc. of IEEE International Conference on Automation and Logistics (2008)

4. Li, X.: A review on integrated process planning and scheduling. Int. J. Manufacturing Research 5(2), 161-180 (2010)

5. Matsusaki, C.T.M., Santos Filho, D.J.: Modeling of distributed collaborative control systems of production systems. In: ABCM Symp. Series in Mechatronics, vol. 2, pp. 345352 (2006)

6. Moriwaki, T.: Multi-functional machine tool. CIRP Annals Manufacturing Technology 57(2), 736-749 (2008)

7. Santos Filho, D.J., Nakamoto, F.Y., Junqueira, F., Miyagi, P.E.: Task Control of Intelligent Transportation Vehicles In Manufacturing Systems. In: Dutra, M.S., Lengerke, O. (eds.) Mechatronics Series 1: Intelligent Transportation Vehicles, vol. 1, pp. 146-169. Bentham Science Publishers, Dubai (2011) 
8. Nakamoto, F.Y.: Resources allocation control in flexible manufacturing systems using the deadlock avoidance method. ABCM Series in Mechatronics, vol. 3, pp. 454-460 (2008)

9. Shin, M.: Self-evolution framework of manufacturing systems based on fractal organization. Computers \& Industrial Engineering 56(3), 1029 (2009)

10. Wiendalhal, H.P., et al.: Changeable manufacturing classification, design and operation. CIRP Annals Manufacturing Technology 56(2), 783-810 (2007)

11. Dorigo, M., Di Caro, G.: The ant colony optimization metaheuristic. In: Corne, D., Dorigo, M., Glover, F. (eds.) New Ideas in Optimization, pp. 11-32. McGraw-Hill, New York (1999)

12. Asato, O.L., Junqueira, F., Santos Filho, D.J., Miyagi, P.E., Araujo Junior, L.O.: Control of Productive Systems with Functional Flexibility Level. In: ETFA 16th IEEE International Conference on Emerging Technologies and Factory Automation. proceedings of ETFA, pp. 1-4. IEEE, Piscataway (2011) 